Kragujevac Journal of Mathematics

Volume 41(1) (2017), Pages 81-92.

\title{
SOME REVERSES OF THE CAUCHY-SCHWARZ AND TRIANGLE INEQUALITIES IN 2-INNER PRODUCT SPACES
}

\author{
MOHSEN ERFANIAN OMIDVAR ${ }^{1}$, HAMID REZA MORADI ${ }^{2}$, \\ SILVESTRU SEVER DRAGOMIR ${ }^{3}$, AND YOEL JE CHO CH, $^{4,}$
}

\begin{abstract}
In this paper, we give some reverses of the Cauchy-Schwarz inequality and triangle inequality in 2-inner product spaces. Applications for determinantal integral inequalities are also provided.
\end{abstract}

\section{INTRODUCTION}

The Cauchy-Schwarz inequality plays an important role in the theory of inner product spaces (see, for instance, $[22,23]$ ), which is one of the classical inequalities. It is well known that, in a semi-inner product space $(\mathscr{X},\langle\cdot, \cdot\rangle)$, the Cauchy-Schwarz inequality has the form

$$
|\langle x, y\rangle|^{2} \leq\langle x, x\rangle\langle y, y\rangle
$$

for all $x, y \in \mathscr{X}$.

In recent years, many authors have studied some related topics such as the reverse of the Cauchy-Schwarz inequality, the triangle and Bessel inequality as well as Grüss inequality (see $[7,10,11,18]$ ). The probably first reverse of the Cauchy-Schwarz inequality for positive real numbers was obtained by Pólya and Szegö in 1925 (see [20, p. 57 and 213-214] and [21, p. 71-72 and 253-255]). Since then, there exist a lot of generalizations of the reverse of the Cauchy-Schwarz inequality. For example, in 2007, Dragomir [6, Chapter 2] contributed much to the reverses of the CauchySchwarz inequality and also similar results for integrals, isotonic functionals as well as generalizations in the setting of inner product spaces are well-studied and understood

Key words and phrases. Cauchy-Schwarz inequality, triangle inequality, 2-inner product, 2-norm. 2010 Mathematics Subject Classification. Primary: 26D15. Secondary: 46C05, 46C50, 26 D10.

Received: June 3, 2016.

Accepted: August 6, 2016. 
(see the book [5]). Some other interesting inequalities for the reverse of the CauchySchwarz inequality can be found in $[8,9,12]$.

In this paper, we continue and complement this research by proving some new reverses of the Cauchy-Schwarz inequality in framework of 2-inner product spaces. Furthermore, as applications, some reverse results for the generalized triangle inequality, i.e., upper bounds for the quantity

$$
(0 \leq) \sum_{i=1}^{n}\left\|x_{i}, z\right\|-\left\|\sum_{i=1}^{n} x_{i}, z\right\|
$$

under various assumptions for the vectors $z, x_{i} \in \mathscr{X}, i \in\{1, \cdots, n\}$, are established and also, some applications for the generalized triangle inequality are given.

\section{Preliminaries}

The concept of 2-normed spaces was introduced by Gähler [16] in 1963. After that, in 1973 and 1977, Diminnie, Gähler and White introduced the concept of 2-inner product spaces $[3,4]$. For more details on 2 -inner product spaces, see $[2,15,17,19]$. A systematic presentation of the recent results related to the theory of 2-inner product spaces as well as an extensive list of the related references can be found in the book [1]. Here we give the basic definitions and the elementary properties of 2-inner product spaces.

Let $\mathscr{X}$ be a linear space of dimension greater than 1 over the field $\mathbb{K}=\mathbb{R}$ of real numbers or the field $\mathbb{K}=\mathbb{C}$ of complex numbers. Suppose that $\langle\cdot, \cdot \mid \cdot\rangle$ is a $\mathbb{K}$-valued function defined on $\mathscr{X} \times \mathscr{X} \times \mathscr{X}$ satisfying the following conditions:

(2I-1) $\langle x, x \mid z\rangle \geq 0$, and $\langle x, x \mid z\rangle=0$ if and only if $x$ and $z$ are linearly dependent;

$(2 \mathrm{I}-2)\langle x, x \mid z\rangle=\langle z, z \mid x\rangle$;

$(2 \mathrm{I}-3)\langle y, x \mid z\rangle=\overline{\langle x, y \mid z\rangle}$;

(2I-4) $\langle\alpha x, y \mid z\rangle=\alpha\langle x, y \mid z\rangle$, for any $\alpha \in \mathbb{K}$;

$(2 \mathrm{I}-5)\left\langle x+x^{\prime}, y \mid z\right\rangle=\langle x, y \mid z\rangle+\left\langle x^{\prime}, y \mid z\right\rangle$.

$\langle\cdot, \cdot \mid \cdot\rangle$ is called a 2-inner product on $\mathscr{X}$ and $(\mathscr{X},\langle\cdot, \cdot \mid \cdot\rangle)$ is called a 2-inner product space (or 2-pre-Hilbert space). Some basic properties of 2 -inner products $\langle\cdot, \cdot \mid \cdot\rangle$ can be immediately obtained as follows:

(P-1) If $\mathbb{K}=\mathbb{R}$, then (2I-3) reduces to $\langle y, x \mid z\rangle=\langle x, y \mid z\rangle$;

$(\mathrm{P}-2)\langle 0, y \mid z\rangle=\langle x, 0 \mid z\rangle=\langle x, y \mid 0\rangle=0$;

(P-3) $\langle x, y \mid \alpha z\rangle=|\alpha|^{2}\langle x, y \mid z\rangle$, for all $x, y, z \in \mathscr{X}$ and $\alpha \in \mathbb{K}$.

Using the above properties, we can prove the Cauchy-Schwarz inequality

$$
|\langle x, y \mid z\rangle|^{2} \leq\langle x, x \mid z\rangle\langle y, y \mid z\rangle .
$$

In any given 2 -inner product space $(\mathscr{X},\langle\cdot, \cdot \mid \cdot\rangle)$, we can define a function $\|\cdot, \cdot\|$ on $\mathscr{X} \times \mathscr{X}$ by

$$
\|x, z\|=\sqrt{\langle x, x \mid z\rangle}
$$


for all $x, z \in \mathscr{X}$. It is easy to see that this function satisfies the following conditions: $(2 \mathrm{~N}-1)\|x, z\| \geq 0$ and $\|x, z\|=0$ if and only if $x$ and $z$ are linearly dependent;

$(2 \mathrm{~N}-2)\|x, z\|=\|z, x\|$

$(2 \mathrm{~N}-3)\|\alpha x, z\|=|\alpha|\|x, z\|$ for any scalar $\alpha \in \mathbb{K}$;

$(2 \mathrm{~N}-4)\left\|x+x^{\prime}, z\right\| \leq\|x, z\|+\left\|x^{\prime}, z\right\|$.

Any function $\|\cdot, \cdot\|$ defined on $\mathscr{X} \times \mathscr{X}$ and satisfying the above conditions is called a 2 -norm on $\mathscr{X}$ and $(\mathscr{X},\|\cdot, \cdot\|)$ is called a linear 2 -normed space. Whenever a 2 -inner product space $(\mathscr{X},\langle\cdot, \cdot \mid \cdot\rangle)$ is given, we consider it as a linear 2 -norm space $(\mathscr{X},\|\cdot, \cdot\|)$ with the 2-norm defined by (2.1).

\section{Some Reverses of the Cauchy-Schwarz Inequality}

First, we have the following.

Theorem 3.1. Let $(\mathscr{X},\langle\cdot, \cdot \mid \cdot\rangle)$ be a 2-inner product space over the real or complex number field $\mathbb{K}$ and $x, y, z \in \mathscr{X}, r_{1}, r_{2}>0$ are such that

$$
r_{1} \leq|\|x, z\|-\|y, z\|| \leq\|x-y, z\| \leq r_{2}
$$

then

$$
\begin{aligned}
\|x, z\|\|y, z\|-|\langle x, y \mid z\rangle| & \leq\|x, z\|\|y, z\|-|\operatorname{Re}\langle x, y \mid z\rangle| \\
& \leq\|x, z\|\|y, z\|-\operatorname{Re}\langle x, y \mid z\rangle \\
& \leq \frac{1}{2}\left(r_{2}^{2}-r_{1}^{2}\right) .
\end{aligned}
$$

Proof. Taking the square in the third inequality in (3.1), we have

$$
\|x, z\|^{2}-2 \operatorname{Re}\langle x, y \mid z\rangle+\|y, z\|^{2} \leq r_{2}^{2},
$$

which is equivalent to

$$
2(\|x, z\|\|y, z\|-\operatorname{Re}\langle x, y \mid z\rangle)+(\|x, z\|-\|y, z\|)^{2} \leq r_{2}^{2} .
$$

Using the first inequality in (3.1), we have

$$
r_{1}^{2} \leq(\|x, z\|-\|y, z\|)^{2} .
$$

Therefore, from (3.3) and (3.4), we have (3.2). This completes the proof.

Corollary 3.1. With all the assumptions of Theorem 3.1, the following holds:

$$
\|x, z\|+\|y, z\|-\|x+y, z\| \leq \sqrt{r_{2}^{2}-r_{1}^{2}} .
$$

Proof. It follows from (3.2) that

$$
\begin{aligned}
(\|x, z\|+\|y, z\|)^{2}-\|x+y, z\|^{2} & =2(\|x, z\|\|y, z\|-\operatorname{Re}\langle x, y \mid z\rangle) \\
& \leq r_{2}^{2}-r_{1}^{2}
\end{aligned}
$$

gives

$$
(\|x, z\|+\|y, z\|)^{2} \leq\|x+y, z\|^{2}+r_{2}^{2}-r_{1}^{2} .
$$


Taking the square root in (3.6) and taking into account that

$$
\sqrt{\alpha+\beta} \leq \sqrt{\alpha}+\sqrt{\beta}
$$

for all $\alpha, \beta \geq 0$, we have the desired inequality (3.5). This completes the proof.

Theorem 3.2. Let $(\mathscr{X},\langle\cdot, \cdot \mid \cdot\rangle)$ be a 2-inner product space over the real or complex number field $\mathbb{K}$. For any $x, y, z \in \mathscr{X}$,

$$
\left\|\frac{x}{\|x, z\|}-\frac{y}{\|y, z\|}, z\right\| \leq r
$$

and

$$
\|x, z\|\|y, z\|-\operatorname{Re}\langle x, y \mid z\rangle \leq \frac{1}{2} r^{2}\|x, z\|\|y, z\|
$$

are equivalent.

Proof. It is obvious by taking the square in (3.7) and performing the required calculations.

Remark 3.1. Since

$$
\begin{aligned}
\|\| y, z\|x-\| x, z\|y, z\| & =\|\| y, z\|(x-y)+(\|y, z\|-\|x, z\|) y, z\| \\
& \leq\|y, z\|\|x-y, z\|+\|\| y, z\|-\| x, z\|\|\|y, z\| \\
& \leq 2\|y, z\|\|x-y, z\|,
\end{aligned}
$$

the sufficient condition for (3.7) to hold is

$$
\|x-y, z\| \leq \frac{r}{2}\|x, z\| .
$$

Theorem 3.3. Let $(\mathscr{X},\langle\cdot, \cdot \mid \cdot\rangle)$ be a 2-inner product space over the real or complex number field $\mathbb{K}$. Then, for any $x, y, z \in \mathscr{X}$ and $p \geq 1$,

$$
\begin{aligned}
0 & \leq\|x, z\|\|y, z\|-|\langle x, y \mid z\rangle| \\
& \leq\|x, z\|\|y, z\|-|\operatorname{Re}\langle x, y \mid z\rangle| \\
& \leq \frac{1}{2}\left\{\begin{array}{l}
\left((\|x, z\|+\|y, z\|)^{2 p}-\|x+y, z\|^{2 p}\right)^{\frac{1}{p}}, \\
\left(\|x-y, z\|^{2 p}-\|x, z\|-\|y, z\| \|^{2 p}\right)^{\frac{1}{p}} .
\end{array}\right.
\end{aligned}
$$

Proof. Firstly, observe that

$$
2(\|x, z\|\|y, z\|-\operatorname{Re}\langle x, y \mid z\rangle)=(\|x, z\|+\|y, z\|)^{2}-\|x+y, z\|^{2} .
$$

Denoting

$$
D:=\|x, z\|\|y, z\|-\operatorname{Re}\langle x, y \mid z\rangle,
$$

we have

$$
2 D+\|x+y, z\|^{2}=(\|x, z\|+\|y, z\|)^{2} .
$$


Taking in (3.9) the power $p \geq 1$ and using the elementary inequality

$$
(a+b)^{p} \geq a^{p}+b^{p}, \quad a, b \geq 0
$$

we have

$$
(\|x, z\|+\|y, z\|)^{2 p}=\left(2 D+\|x+y, z\|^{2}\right)^{p} \geq 2^{p} D^{p}+\|x+y, z\|^{2 p} .
$$

This implies that

$$
D^{p} \leq \frac{1}{2^{p}}\left((\|x, z\|+\|y, z\|)^{2 p}-\|x+y, z\|^{2 p}\right),
$$

which is clearly equivalent to the first branch of the third inequality in (3.8).

With the above notation, we also have

$$
2 D+(\|x, z\|-\|y, z\|)^{2}=\|x-y, z\|^{2} .
$$

Taking the power $p \geq 1$ in (3.11) and using the inequality (3.10), we have

$$
\|x-y, z\|^{2 p} \geq 2^{p} D^{p}+|\|x, z\|-\|y, z\||^{2 p}
$$

and so we have the last part of (3.8). This completes the proof.

We state the following result that provides an invariant property for the constant in the Cauchy-Schwarz inequality.

Theorem 3.4. Let $(\mathscr{X},\langle\cdot, \cdot \mid \cdot\rangle)$ be a 2-inner product space over the real or complex number field $\mathbb{K}$. For any $x, y, z \in \mathscr{X}$ and $\lambda \in \mathbb{K}$,

$$
\|x, z\|^{2}\|y, z\|^{2}-|\langle x, y \mid z\rangle|^{2}=\|x-\lambda y, z\|^{2}\|y, z\|^{2}-|\langle x-\lambda y, y \mid z\rangle|^{2} .
$$

Proof. By properties of 2 -inner product, it follows that, for any $x, y, z \in \mathscr{X}$ and $\lambda \in \mathbb{K}$,

$$
\begin{aligned}
& \|x-\lambda y, z\|^{2}\|y, z\|^{2}-|\langle x-\lambda y, y \mid z\rangle|^{2} \\
= & \left(\|x, z\|^{2}-2 \operatorname{Re}(\bar{\lambda}\langle x, y \mid z\rangle)+|\lambda|^{2}\|y, z\|^{2}\right)\|y, z\|^{2} \\
& -\left|\langle x, y \mid z\rangle-\lambda\|y, z\|^{2}\right|^{2} \\
= & \|x, z\|^{2}\|y, z\|^{2}-2\|y, z\|^{2} \operatorname{Re}(\bar{\lambda}\langle x, y \mid z\rangle)+|\lambda|^{2}\|y, z\|^{4} \\
& -|\langle y, x \mid z\rangle|^{2}+2\|y, z\|^{2} \operatorname{Re}(\bar{\lambda}\langle x, y \mid z\rangle)-|\lambda|^{2}\|y, z\|^{4} \\
= & \|y, z\|^{2}\|x, z\|^{2}-|\langle y, x \mid z\rangle|^{2} .
\end{aligned}
$$

This completes the proof.

Corollary 3.2. Let $(\mathscr{X},\langle\cdot, \cdot \mid \cdot\rangle)$ be a 2-inner product space over the real or complex number field $\mathbb{K}$. For any $x, y, z \in \mathscr{X}$ and $\lambda \in \mathbb{K}$,

$$
\|x, z\|^{2}\|y, z\|^{2}-|\langle x, y \mid z\rangle|^{2} \leq\|x-\lambda y, z\|^{2}\|y, z\|^{2} .
$$

The equalities holds in (3.12) if and only if $\langle x, y \mid z\rangle=\lambda\|y, z\|^{2}$.

For two parameters, we can get the following. 
Theorem 3.5. Let $(\mathscr{X},\langle\cdot, \cdot \mid \cdot\rangle)$ be a 2-inner product space over the real or complex number field $\mathbb{K}$. For any $x, y, z \in \mathscr{X}$ and $\lambda, \mu \in \mathbb{K}$,

$$
\begin{aligned}
& \left(\|x, z\|^{2}\|y, z\|^{2}-|\langle x, y \mid z\rangle|^{2}\right)|\mu-\lambda|^{2} \\
= & \|x-\lambda y, z\|^{2}\|x-\mu y, z\|^{2}-|\langle x-\lambda y, x-\mu y \mid z\rangle|^{2} .
\end{aligned}
$$

Proof. Denote $w:=x-\lambda y$. Using some properties of a 2-inner product, we have

$$
\begin{aligned}
|\langle x-\lambda y, x-\mu y \mid z\rangle|^{2}= & |\langle w, x-\mu y \mid z\rangle|^{2} \\
= & |\langle w, x-\lambda y+(\lambda-\mu) y \mid z\rangle|^{2} \\
= & |\langle w, w+(\lambda-\mu) y \mid z\rangle|^{2} \\
= & \left|\|w, z\|^{2}+\overline{(\lambda-\mu)}\langle w, y \mid z\rangle\right|^{2} \\
= & \|w, z\|^{4}+2\|w, z\|^{2} \operatorname{Re}(\lambda-\mu) \overline{\langle w, y \mid z\rangle} \\
& +|\lambda-\mu|^{2}|\langle w, y \mid z\rangle|^{2} \\
= & \|w, z\|^{4}+2\|w, z\|^{2} \operatorname{Re}((\lambda-\mu) \overline{\langle w, y \mid z\rangle}) \\
& +|\lambda-\mu|^{2}\|w, z\|^{2}\|y, z\|^{2} \\
& -|\lambda-\mu|^{2}\left(\|w, z\|^{2}\|y, z\|^{2}-|\langle w, y \mid z\rangle|^{2}\right) .
\end{aligned}
$$

Observe also that

$$
\begin{aligned}
& \|w, z\|^{4}+2\|w, z\|^{2} \operatorname{Re}((\lambda-\mu) \overline{\langle w, y \mid z\rangle})+|\lambda-\mu|^{2}\|w, z\|^{2}\|y, z\|^{2} \\
= & \|w, z\|^{2}\left(\|w, z\|^{2}+2 \operatorname{Re}(\overline{(\lambda-\mu) \overline{\langle w, y \mid z\rangle}})+|\lambda-\mu|^{2}\|y, z\|^{2}\right) \\
= & \|w, z\|^{2}\|w+(\lambda-\mu) y, z\|^{2} \\
= & \|x-\lambda y, z\|^{2}\|x-\mu y, z\|^{2} .
\end{aligned}
$$

Therefore, from (3.14) and (3.15), we have the desired result (3.13). This completes the proof.

Corollary 3.3. Let $(\mathscr{X},\langle\cdot, \cdot \mid \cdot\rangle)$ be a 2-inner product space over the real or complex number field $\mathbb{K}$. For any $x, y, z \in \mathscr{X}$ and $\lambda, \mu \in \mathbb{K}$,

$$
\|x, z\|^{2}\|y, z\|^{2}-|\langle x, y \mid z\rangle|^{2} \leq \frac{1}{|\mu-\lambda|^{2}}\|x-\lambda y, z\|^{2}\|x-\mu y, z\|^{2} .
$$

As an application of Theorem 3.5, we have the following.

Proposition 3.1. Let $(\mathscr{X},\langle\cdot, \cdot \mid \cdot\rangle)$ be a 2-inner product space over the real or complex number field $\mathbb{K}$. Then, for all $x, y, z, e \in \mathscr{X}$ with $\|e, z\|=1$ and $\lambda, \mu, \gamma, \eta \in \mathbb{K}$ with $\lambda \neq \mu$ and $\gamma \neq \eta$,

$$
|\langle x, y \mid z\rangle-\langle x, e \mid z\rangle\langle e, y \mid z\rangle|
$$




$$
\begin{aligned}
\leq & \frac{1}{|\lambda-\mu||\gamma-\eta|}(\|x-\lambda e, z\|\|x-\mu e, z\|\|y-\gamma e, z\|\|y-\eta e, z\| \\
& -|\langle x-\lambda e, x-\mu e \mid z\rangle||\langle y-\gamma e, y-\eta e \mid z\rangle|) \\
\leq & \frac{1}{|\lambda-\mu||\gamma-\eta|}(\|x-\lambda e, z\|\|x-\mu e, z\|\|y-\gamma e, z\|\|y-\eta e, z\|) .
\end{aligned}
$$

Proof. Applying the Cauchy-Schwarz inequality for the vectors $x-\langle x, e \mid z\rangle e$ and $y-\langle y, e \mid z\rangle e$ and taking into account that

$$
\langle x-\langle x, e \mid z\rangle e, y-\langle y, e \mid z\rangle e \mid z\rangle=\langle x, y \mid z\rangle-\langle x, e \mid z\rangle\langle e, y \mid z\rangle
$$

and

$$
\|x-\langle x, e \mid z\rangle e, z\|^{2}=\|x, z\|^{2}-|\langle x, e \mid z\rangle|^{2}
$$

and

we have

$$
\|y-\langle y, e \mid z\rangle e, z\|^{2}=\|y, z\|^{2}-|\langle y, e \mid z\rangle|^{2}
$$

$$
\begin{aligned}
& |\langle x, y \mid z\rangle-\langle x, e \mid z\rangle\langle e, y \mid z\rangle| \\
\leq & \left(\|x, z\|^{2}-|\langle x, e \mid z\rangle|^{2}\right)^{\frac{1}{2}}\left(\|y, z\|^{2}-|\langle y, e \mid z\rangle|^{2}\right)^{\frac{1}{2}}
\end{aligned}
$$

for any $x, y, z, e \in \mathscr{X}$ with $\|e, z\|=1$.

From (3.13), it follows that

$$
\begin{aligned}
& \left(\|x, z\|^{2}-|\langle x, e \mid z\rangle|^{2}\right)^{\frac{1}{2}} \\
= & \frac{1}{|\mu-\lambda|}\left(\|x-\lambda e, z\|^{2}\|x-\mu e, z\|^{2}-|\langle x-\lambda e, x-\mu e \mid z\rangle|^{2}\right)^{\frac{1}{2}}
\end{aligned}
$$

and

$$
\begin{aligned}
& \left(\|y, z\|^{2}-|\langle y, e \mid z\rangle|^{2}\right)^{\frac{1}{2}} \\
= & \frac{1}{|\gamma-\eta|}\left(\|y-\gamma e, z\|^{2}\|y-\eta e, z\|^{2}-|\langle y-\gamma e, y-\eta e \mid z\rangle|^{2}\right)^{\frac{1}{2}},
\end{aligned}
$$

for any $x, y, z, e \in \mathscr{X}$ with $\|e, z\|=1$ and $\lambda, \mu, \gamma, \eta \in \mathbb{K}$ with $\lambda \neq \mu$ and $\gamma \neq \eta$.

Now, if we multiply (3.18) with (3.19), then we have

$$
\begin{aligned}
& \left(\|x, z\|^{2}-|\langle x, e \mid z\rangle|^{2}\right)^{\frac{1}{2}}\left(\|y, z\|^{2}-|\langle y, e \mid z\rangle|^{2}\right)^{\frac{1}{2}} \\
\leq & \frac{1}{|\mu-\lambda||\gamma-\eta|} \\
& \times\left(\|x-\lambda e, z\|^{2}\|x-\mu e, z\|^{2}|\langle x-\lambda e, x-\mu e \mid z\rangle|^{2}\right)^{\frac{1}{2}} \\
& \times\left(\|y-\gamma e, z\|^{2}\|y-\eta e, z\|^{2}|\langle y-\gamma e, y-\eta e \mid z\rangle|^{2}\right)^{\frac{1}{2}} .
\end{aligned}
$$

Further, if we use the elementary inequality

$$
\left(a^{2}-b^{2}\right)^{\frac{1}{2}}\left(c^{2}-d^{2}\right)^{\frac{1}{2}} \leq a c-b d
$$


for all $a \geq b \geq 0$ and $c \geq d \geq 0$, then we also have

$$
\begin{aligned}
& \left(\|x-\lambda e, z\|^{2}\|x-\mu e, z\|^{2}|\langle x-\lambda e, x-\mu e \mid z\rangle|^{2}\right)^{\frac{1}{2}} \\
& \times\left(\|y-\gamma e, z\|^{2}\|y-\eta e, z\|^{2}|\langle y-\gamma e, y-\eta e \mid z\rangle|^{2}\right)^{\frac{1}{2}} \\
\leq & \|x-\lambda e, z\|\|x-\mu e, z\|\|y-\gamma e, z\|\|y-\eta e, z\| \\
& -|\langle x-\lambda e, x-\mu e \mid z\rangle||\langle y-\gamma e, y-\eta e \mid z\rangle| .
\end{aligned}
$$

Finally, using (3.17), (3.20) and (3.21), we have the second inequality (3.16). This completes the proof.

\section{Some Reverses of the Triangle Inequality}

In this section, we give some reverses of the triangle inequality.

Theorem 4.1. Let $(\mathscr{X},\langle\cdot, \cdot \mid \cdot\rangle)$ be a 2-inner product space over the real or complex number field $\mathbb{K}$. Let $z, x_{i}, \in \mathscr{X}, i \in\{1, \ldots, n\}$, and $r_{i j}>0$ for each $1 \leq i \leq j \leq n$ be such that

$$
0 \leq\left\|x_{i}, z\right\|\left\|x_{j}, z\right\|-\operatorname{Re}\left\langle x_{i}, x_{j} \mid z\right\rangle \leq r_{i j} .
$$

Then the following quadratic reverse of the triangle inequality holds:

$$
\left(\sum_{i=1}^{n}\left\|x_{i}, z\right\|\right)^{2} \leq\left\|\sum_{i=1}^{n} x_{i}, z\right\|^{2}+2 \sum_{1 \leq i \leq j \leq n} r_{i j} .
$$

The case of the equality holds in (4.2) if and only if it holds in (4.1) for each $i, j$ with $1 \leq i \leq j \leq n$.

Proof. Observe that

$$
\begin{aligned}
\left(\sum_{i=1}^{n}\left\|x_{i}, z\right\|\right)^{2}-\left\|\sum_{i=1}^{n} x_{i}, z\right\|^{2}= & \sum_{i, j=1}^{n}\left\|x_{i}, z\right\|\left\|x_{j}, z\right\|-\left\langle\sum_{i=1}^{n} x_{i}, \sum_{j=1}^{n} x_{j} \mid z\right\rangle \\
= & \sum_{i, j=1}^{n}\left\|x_{i}, z\right\|\left\|x_{j}, z\right\|-\sum_{i, j=1}^{n} \operatorname{Re}\left\langle x_{i}, x_{j} \mid z\right\rangle \\
= & \sum_{i, j=1}^{n}\left(\left\|x_{i}, z\right\|\left\|x_{j}, z\right\|-\operatorname{Re}\left\langle x_{i}, x_{j} \mid z\right\rangle\right) \\
= & \sum_{1 \leq i \leq j \leq n}\left(\left\|x_{i}, z\right\|\left\|x_{j}, z\right\|-\operatorname{Re}\left\langle x_{i}, x_{j} \mid z\right\rangle\right) \\
& +\sum_{1 \leq j \leq i \leq n}\left(\left\|x_{i}, z\right\|\left\|x_{j}, z\right\|-\operatorname{Re}\left\langle x_{i}, x_{j} \mid z\right\rangle\right) \\
= & 2 \sum_{1 \leq i \leq j \leq n}\left(\left\|x_{i}, z\right\|\left\|x_{j}, z\right\|-\operatorname{Re}\left\langle x_{i}, x_{j} \mid z\right\rangle\right) .
\end{aligned}
$$


Using the condition (4.1), we have

$$
\sum_{1 \leq i \leq j \leq n}\left(\left\|x_{i}, z\right\|\left\|x_{j}, z\right\|-\operatorname{Re}\left\langle x_{i}, x_{j} \mid z\right\rangle\right) \leq \sum_{1 \leq i \leq j \leq n} r_{i j}
$$

and, by (4.3), we have the desired inequality (4.2).

The case of the equality is obvious by the identity (4.3) and we omit the details. This completes the proof.

Remark 4.1. From (4.2), one may deduce the coarser inequality that might be useful in some applications:

$$
0 \leq \sum_{i=1}^{n}\left\|x_{i}, z\right\|-\left\|\sum_{i=1}^{n} x_{i}, z\right\| \leq \sqrt{2}\left(\sum_{1 \leq i \leq j \leq n} r_{i j}\right)^{\frac{1}{2}}
$$

Theorem 4.2. Let $(\mathscr{X},\langle\cdot, \cdot \mid \cdot\rangle)$ be a 2-inner product space over the real or complex number field $\mathbb{K}$. Let $z, x_{i}, \in \mathscr{X}, i \in\{1, \ldots, n\}$, and $r>0$ be such that

$$
\left\|x_{i}-x_{j}, z\right\| \leq r
$$

for each $1 \leq i \leq j \leq n$. Then

$$
\left(\sum_{i=1}^{n}\left\|x_{i}, z\right\|\right)^{2} \leq\left\|\sum_{i=1}^{n} x_{i}, z\right\|^{2}+\frac{n(n-1)}{2} r^{2} .
$$

The case of the equality holds in (4.5) if and only if

$$
\left\|x_{i}, z\right\|\left\|x_{j}, z\right\|-\operatorname{Re}\left\langle x_{i}, x_{j} \mid z\right\rangle=\frac{1}{2} r^{2},
$$

for each $1 \leq i \leq j \leq n$.

Proof. The inequality (4.4) is obviously equivalent to

$$
\left\|x_{i}, z\right\|^{2}+\left\|x_{j}, z\right\|^{2} \leq 2 \operatorname{Re}\left\langle x_{i}, x_{j} \mid z\right\rangle+r^{2},
$$

for each $1 \leq i \leq j \leq n$. Since

$$
2\left\|x_{i}, z\right\|\left\|x_{j}, z\right\| \leq\left\|x_{i}, z\right\|^{2}+\left\|x_{j}, z\right\|^{2},
$$

for each $1 \leq i \leq j \leq n$, we have

$$
\left\|x_{i}, z\right\|\left\|x_{j}, z\right\|-\operatorname{Re}\left\langle x_{i}, x_{j} \mid z\right\rangle \leq \frac{1}{2} r^{2},
$$

for each $1 \leq i \leq j \leq n$.

Applying Theorem 4.1 for $r_{i j}:=\frac{1}{2} r^{2}$ and taking into account that

$$
\sum_{1 \leq i \leq j \leq n} r_{i j}=\frac{n(n-1)}{4} r^{2}
$$

we deduce the desired inequality (4.5). The case of the equality is also obvious by the Theorem 4.1 and we omit the details. This completes the proof. 


\section{Applications for Determinantal Integral Inequalities}

Let $(\Omega, \Sigma, \mu)$ be a measure space consisting of a set $\Omega$, a $\sigma$-algebra $\Sigma$ of subsets of $\Omega$ and a countably additive and positive measure $\mu$ on $\Sigma$ with values in $\mathbb{R} \cup\{\infty\}$. Denote by $L_{\rho}^{2}(\Omega)$ the Hilbert space of all real-valued functions $f$ defined on $\Omega$ that are 2 - $\rho$-integrable on $\Omega$, i.e.

$$
\int_{\Omega} \rho(s)|f(s)|^{2} d \mu(s)<\infty
$$

where $\rho: \Omega \rightarrow[0, \infty)$ is a measurable function on $\Omega$.

We can introduce the following 2 -inner product on $L_{\rho}^{2}(\Omega)$ by the formula

$$
\langle f, g \mid h\rangle_{\rho}:=\frac{1}{2} \int_{\Omega} \int_{\Omega} \rho(s) \rho(t)\left|\begin{array}{ll}
f(s) & f(t) \\
h(s) & h(t)
\end{array}\right|\left|\begin{array}{ll}
g(s) & g(t) \\
h(s) & h(t)
\end{array}\right| d \mu(s) d \mu(t),
$$

where, by

$$
\left|\begin{array}{ll}
f(s) & f(t) \\
h(s) & h(t)
\end{array}\right|
$$

we denote the determinant of the matrix

$$
\left|\begin{array}{ll}
g(s) & g(t) \\
h(s) & h(t)
\end{array}\right|
$$

generating the 2-norm on $L_{\rho}^{2}(\Omega)$ expressed by

$$
\|f, h\|_{\rho}:=\left(\frac{1}{2} \int_{\Omega} \int_{\Omega} \rho(s) \rho(t)\left|\begin{array}{ll}
f(s) & f(t) \\
h(s) & h(t)
\end{array}\right| d \mu(s) d \mu(t)\right)^{\frac{1}{2}} .
$$

A simple calculation with integrals reveals that

$$
\langle f, g \mid h\rangle_{\rho}=\left|\begin{array}{ll}
\int_{\Omega} \rho f g d \mu & \int_{\Omega} \rho f h d \mu \\
\int_{\Omega} \rho g h d \mu & \int_{\Omega} \rho h^{2} d \mu
\end{array}\right|
$$

and

$$
\|f, h\|_{\rho}=\left|\begin{array}{ll}
\int_{\Omega} \rho f^{2} d \mu & \int_{\Omega} \rho f h d \mu \\
\int_{\Omega} \rho f h d \mu & \int_{\Omega} \rho h^{2} d \mu
\end{array}\right|^{\frac{1}{2}}
$$

where, for simplicity, instead of $\int_{\Omega} \rho(s) f(s) g(s) d \mu(s)$, we have written $\int_{\Omega} \rho f g d \mu$ (see, e.g. [13] or [14]).

Using the representations (5.1), (5.2) and the Theorem 4.1, one may state interesting determinantal integral inequality, as follows. 
Proposition 5.1. Let $f_{1}, \ldots, f_{n}, g, h \in L_{\rho}^{2}(\Omega)$, where $\rho: \Omega \rightarrow[0, \infty)$ is a measurable function on $\Omega$ and $r_{i j}>0$ such that

$$
\left|\begin{array}{cc}
\int_{\Omega} \rho\left(f_{i}-f_{j}\right)^{2} d \mu & \int_{\Omega} \rho\left(f_{i}-f_{j}\right) h d \mu \\
\int_{\Omega} \rho\left(f_{i}-f_{j}\right) h d \mu & \int_{\Omega} \rho h^{2} d \mu
\end{array}\right|^{\frac{1}{2}} \leq r
$$

for each $1 \leq i \leq j \leq n$, then

$$
\left(\sum_{i=1}^{n}\left|\begin{array}{ll}
\int_{\Omega} \rho f_{i}^{2} d \mu & \int_{\Omega} \rho f_{i} h d \mu \\
\int_{\Omega} \rho f_{i} h d \mu & \int_{\Omega} \rho h^{2} d \mu
\end{array}\right|\right)^{\frac{1}{2}} \leq\left|\begin{array}{ll}
\sum_{i=1}^{n} \int_{\Omega} \rho f_{i}^{2} d \mu & \sum_{i=1}^{n} \int_{\Omega} \rho f_{i} h d \mu \\
\sum_{i=1}^{n} \int_{\Omega} \rho f_{i} h d \mu & \sum_{i=1}^{n} \int_{\Omega} \rho h^{2} d \mu
\end{array}\right|+2 \sum_{1 \leq i \leq j \leq n} r_{i j} .
$$

Proof. The proof follows by Theorem 4.1, applied for the 2-inner product $\langle\cdot, \cdot \mid \cdot\rangle_{\rho}$ and we omit the details.

Similar determinantal integral inequalities may be stated if one uses the other results for 2-inner products obtained above, but we do not present them here.

Acknowledgements. The authors would like to express their cordial thanks to the referee for several valuable suggestions. Yeol Je Cho was supported by Basic Science Research Program through the National Research Foundation of Korea (NRF) funded by the Ministry of Science, ICT and future Planning (2014R1A2A2A01002100).

\section{REFERENCES}

[1] Y. J. Cho, C. S. Lin, S. S. Kim and A. Misiak, Theory of 2-inner product spaces, Nova Science Publishers Inc., New York, 2001.

[2] Y. J. Cho, M. Matić and J. E. Pečarić, On Gram's determinant in 2-inner product spaces, J. Korean Math. Soc. 38 (2001), 1125-1156.

[3] C. R. Diminnie, S. Gähler and A. White, A: 2-inner product spaces, Demonst. Math. 6 (1973), $525-536$.

[4] C. R. Diminnie, S. Gähler and A. White, 2-inner product spaces II, Demonst. Math. 10 (1977), 169-188.

[5] S. S. Dragomir, Advances in Inequalities of the Schwarz, Grüss, and Bessel Type in Inner Product Spaces, Nova Science Publishers, Inc. New York, 2005.

[6] S. S. Dragomir, Advances in Inequalities of the Schwarz, Triangle and Heisenberg Type in Inner Product Spaces, Nova Science Publishers Inc., New York, 2007.

[7] S. S. Dragomir, A note on Bessel's inequality, Austral. Math. Soc. Gaz. 28 (2001), 246-248.

[8] S. S. Dragomir, A potpourri of Schwarz related inequalities in inner product spaces (I), J. Ineq. Pure \& Appl. Math. 6 (2005).

[9] S. S. Dragomir, Further reverses of the Schwarz inequality in inner product spaces, East Asian Math. J. 22 (2006), 1-15.

[10] S. S. Dragomir, On Bessel and Grüss inequalities for orthonormal families in inner product spaces, Bull. Austral. Math. Soc. 69 (2004), 327-340.

[11] S. S. Dragomir, Reverses of Schwarz inequality in inner product spaces with applications, Math. Nachr. 288 (2015), 730-742. 
[12] S. S. Dragomir, Reverses of Schwarz, triangle and Bessel inequalities in inner product spaces, J. Inequal. Pure \& Appl. Math. 5 (2004), 1-10.

[13] S. S. Dragomir, Y. J. Cho, S. S. Kim, and Y. H. Kim, On Bessel's and Grüss' inequalities for orthornormal families in 2-inner product spaces and applications, RGMIA research report collection 6(4) (2003).

[14] S. S. Dragomir, Y. J. Cho, S. S. Kim, and Y. H. Kim, Some Boas-Bellman type inequalities in 2-inner product spaces, J. Ineq. Pure \& Appl. Math. 6(2) (2005), Article ID 55.

[15] R. W. Freese, S. S. Dragomir, Y. J. Cho and S. S. Kim, Some componions of Grüss inequality in 2-inner product space and applications for determinantal integral inequalities, Commun. Korean Math. Soc. 20 (2005), 487-503.

[16] S. Gähler, Lineare 2-normierte Räume, Math. Nachr. 28 (1965), 1-43.

[17] Z. Lewandowska, Bounded 2-linear operators on 2-normed sets, Gals. Mat. 39(59) (2004), 303314.

[18] H. R. Moradi, M. E. Omidvar and S. S. Dragomir, Some inequalities for absolute value in Hilbert $C^{*}$-modules, RGMIA Research Report Collection 18 (2015), Article ID 132.

[19] A. Najati, M. M. Saem and J. H. Bae, Generalized Dunkl-Williams inequality in 2-inner product spaces, J. Inequal. Appl. 1 (2013), 1-8.

[20] G. Pólya and G. Szegö, Aufgaben und Lehrsẗze aus der Analysis. Band 1: Reihen, Integralrechnung, Funktionentheorie, (in German), 4th Ed., Springer-Verlag, Berlin, 1970 (original version: Julius Springer Berlin, 1925).

[21] G. Pólya and G. Szegö, Problems and theorems in analysis, Vol. I: Series, integral calculus, theory of functions, Translated from the German by D. Aeppli Die Grundlehren der Mathematischen Wissenschaften, Band 193. Springer-Verlag, New York-Berlin, 1972.

[22] T. M. Rassias, Survey on Classical Inequalities, Kluwer Academic Publishers, Dordrecht, Boston, London, 2000.

[23] T. M. Rassias, Functional Equations, Inequalities and Applications, Kluwer Academic Publishers, Dordrecht, Boston, London, 2003.

${ }^{1}$ Department Of Mathematics,

MashHad Branch, Islamic Azad University,

MASHHAD, IRAN

E-mail address: erfanian@mshdiau.ac.ir

${ }^{2}$ Department Of Mathematics,

Mashhad Branch, Islamic Azad University,

MASHHAD, IRAN

E-mail address: hrmoradi@mshdiau.ac.ir

${ }^{3}$ Department Of Mathematics,

College of Engineering and Science, Victoria University,

P.O. Box 14428, Melbourne City, MC 8001, Australia.

E-mail address: sever.dragomir@vu.edu.au

${ }^{4}$ Department of Mathematics Education and the Rins,

Gyeongsang National University,

Chinju 660-701, Korea

${ }^{5}$ Department of Mathematics,

King AbDulaziz University,

JEDDAH 21589, SAUdi Arabia

E-mail address: yjcho@nongae.gsnu.ac.kr 